\title{
Contemporary Strategies to Manage High Blood Pressure in Patients with Coexistent Resistant Hypertension and Heart Failure With Reduced Ejection Fraction
}

\author{
Katherine Lang • Erik H. Van Iterson (D) · Luke J. Laffin
}

Received: October 12, 2020 / Published online: November 17, 2020

(c) The Author(s) 2020

\begin{abstract}
Resistant hypertension (RH) represents an advanced subtype of hypertension that is complex to diagnose and treat. Compared with general hypertension, $\mathrm{RH}$ increases the risk patients will develop more advanced cardiovascular complications, including heart failure with reduced ejection fraction (HFrEF). As expected, the prevalence of $\mathrm{RH}$ has increased since the introduction of lower blood pressure targets included in the recent 2017 American blood pressure guidelines. The array of pharmacotherapies available to treat both hypertension and HFrEF has also expanded within the
\end{abstract}

past decade. However, the efficacy of these cutting-edge pharmacotherapies has not come without a more advanced understanding of the important adjunct role non-pharmacological therapies play in helping with the management of both hypertension and HFrEF. In this review, we provide a summary of the latest pharmacological and non-pharmacological strategies that can be used to initiate treatment and optimize long-term blood pressure control in patients with coexistent RH and HFrEF.

Keywords: Heart failure; Heart failure with reduced ejection fraction; Hypertension; Resistant hypertension

\section{K. Lang}

Case Western Reserve University School of

Medicine, Cleveland, $\mathrm{OH}$, USA

E. H. Van Iterson $(\varangle) \cdot$ L. J. Laffin

Section of Preventive Cardiology and

Rehabilitation, Department of Cardiovascular

Medicine, Cleveland Clinic Foundation, Cleveland,

$\mathrm{OH}$, USA

e-mail: vanitee@ccf.org 


\section{Key Summary Points}

Resistant hypertension (RH) represents an advanced subtype of hypertension that is complex to diagnose, treat, and increases the risk patients develop advanced cardiovascular complications, such as heart failure with reduced ejection fraction (HFrEF).

As the number of patients with RH continues to rise in the United States, so too does the prevalence of patients with HFrEF and also those with coexistent RH and HFrEF.

There is no randomized controlled clinical trial-based evidence available to guide the use of pharmacotherapies for the optimal management of BP specifically in patients with coexistent RH and HFrEF.

In this review, we summarize the latest pharmacological and non-

pharmacological interventions that are available for potentially optimizing BP control in patients with coexistent $\mathrm{RH}$ and HFrEF.

Non-pharmacological therapies, including exercise training, when individually prescribed based on the model established in HFrEF is medically reasonable and can be expected to contribute to the effective management of both resting and ambulatory BP in patients with coexistent RH and HFrEF.

Cutting-edge treatment options, including small interfering RNAs, renal denervation, and baroreflex activation therapy, are currently under investigation. As higherquality data become available from randomized controlled clinical trials, it can be expected that the continued evolution of BP treatment guideline recommendations will ultimately allow for the advanced development of individualized treatment plans that can durably maintain BP below guidelinerecommended targets.

\section{DIGITAL FEATURES}

This article is published with digital features to facilitate understanding of the article. You can access the digital features on the article's associated Figshare page. To view digital features for this article go to https://doi.org/10.6084/m9. figshare. 13160822

\section{INTRODUCTION}

Hypertension is a world-wide public health concern that not only persists as a key risk factor for the development of more advanced and chronic cardiovascular conditions, such as ischemic heart disease and heart failure (HF), unmanaged high blood (BP) pressure also increases the risk of both hospitalizations and mortality [1-3]. As expected, these concerns have recently been amplified as the estimated prevalence of high BP requiring treatment in adults aged 20 years and older residing in the United States (US) took a major uptick following the release of the 2017 American Heart Association (AHA) and American College of Cardiology (ACC) BP guidelines [4, 5]. The decision to move towards more aggressive $\mathrm{BP}$ targets for both initiating treatment and goal setting largely came as a result of powerful data generated from the Systolic Blood Pressure Intervention Trial (SPRINT) [3]. Published in 2015, SPRINT demonstrated using a randomized controlled and open-label study design that in non-diabetic patients, aggressive management of hypertension to a systolic pressure goal of $<120 \mathrm{mmHg}$ lowers rates of adverse cardiovascular events, predominantly those associated with new-onset HF, as well as all-cause mortality [3]. The strength of the SPRINT evidence confirms how important it is to promptly diagnose, initiate therapy, and aggressively manage hypertension in both primary and secondary prevention settings as a long-term strategy that can appreciably lessen the risk of adverse clinical outcomes.

In addition to leading to an increase in the overall prevalence of hypertension, the recent lowering of BP targets has also led to a higher number of patients diagnosed with resistant 
hypertension (RH). This is generally defined as an above target BP in patients taking three or more BP-lowering medications at maximally tolerated doses, including a diuretic appropriately dosed for renal function, as well as, ideally, a dihydropyridine calcium channel blocker (CCB) and a renin-angiotensin-aldosterone system (RAAS) blocker. However, because not all patients with RH strictly meet such criteria, this has led to the identification of subclasses of $\mathrm{RH}$, including those with either controlled $\mathrm{RH}$ or refractory hypertension. Patients with socalled controlled $\mathrm{RH}$ are able to maintain $\mathrm{BP}$ at or below target while taking four or more BPlowering medications [6]. Alternatively, the newer subcategory of RH termed refractory hypertension describes individuals who demonstrate persistent uncontrolled BP while taking five or more BP-lowering medications, including a mineralocorticoid receptor antagonist (MRA), such as spironolactone, and a longacting thiazide-type diuretic, such as chlorthalidone [7].

Hypertension has long been considered a major risk factor for $\mathrm{HF}$, although typically discussed with less urgency as compared to links associated with coronary artery disease and myocardial ischemia $[1,2,8-10]$. To date, however, there remains a paucity of large-scale randomized controlled clinical trial-based evidence to properly guide the use of pharmacotherapies for the optimal management of BP specifically in patients with coexistent $\mathrm{RH}$ and HF with reduced ejection fraction (HFrEF) [8]. Therefore, given the association between uncontrolled hypertension and an increased risk of developing advanced cardiovascular complications, including HFrEF [1-3], it is crucial to identify both pharmacological and nonpharmacological therapies that can be used right now for optimizing BP control in patients with coexistent RH and HFrEF.

In this review, we summarize and discuss the latest pharmacological and non-pharmacological interventions currently available for initiating treatment and managing long-term BP control in patients with coexistent $\mathrm{RH}$ and HFrEF.

This article is based on previously conducted studies and does not contain any new studies with human participants or animals performed by any of the authors.

\section{Epidemiology}

More than two out of every five adults residing in the US are reported to demonstrate high $\mathrm{BP}$ requiring treatment [5]. It follows that of those who are treated, uncontrolled BP can persist in a select number of patients where it is appropriate to diagnose $\mathrm{RH}$. However, deciding which patients with uncontrolled BP have true $\mathrm{RH}$ can be a complex process. This is because other common clinical scenarios, such as pseudo-resistant hypertension possibly related to medication non-adherence or white coat hypertension, must first be ruled out as possible causes for uncontrolled BP [11].

Despite the difficulties involved in diagnosing $\mathrm{RH}$, prevalence reported in the US prior to the $2017 \mathrm{BP}$ guideline changes estimated that nearly $13 \%$ of adults treated for hypertension are of the RH subtype [12]. This is a number that represents more than a twofold rise in estimated prevalence since the 1990s [12]. Although more recent estimates have placed the number of patients with $\mathrm{RH}$ closer to $16 \%$, this upward bump of nearly 3\% since being reported in 2012 can be largely explained by the aforementioned recent $\mathrm{BP}$ target changes $[12,13]$.

HF is a global public health epidemic. Current reports estimate there are more than 6.2 million patients with HF residing in the US, approximately half of which are of the HFrEF phenotype [5]. The accompanying healthcare costs associated with managing care for these patients is currently believed to exceed $\$ 30.7$ billion annually, a number that is projected to reach at least $\$ 69.7$ billion by the year 2030 [5]. The anticipated rise in both the number of patients and associated healthcare costs can be largely explained by the vast increase in the projected number of adults over the age of 65 by the year 2030 coupled with an already-known understanding that the risk of HF markedly increases beyond 65 years of age $[5,14]$. The implications of these forthcoming estimates are grave since elderly aged patients with HF are at 
the highest risk levels for cardiovascular-related hospitalizations and death $[5,15,16]$.

While a limited number of data are available to confirm a causal link between RH and HFrEF, several studies have reported on the coexistence of RH and HFrEF. For example, it has been estimated that approximately $15 \%$ of Chinese patients with HFrEF (defined as LVEF $<50 \%$ ) demonstrate overlapping $\mathrm{RH}$, whereas this number drops to $11 \%$ in those with $\mathrm{HF}$ and preserved ejection fraction (HFpEF) [17]. Others have also suggested that the presence of RH not only increases the risk of HF but also the likelihood of adverse cardiovascular events, such as myocardial infarction and/or stroke [18, 19]. Thus, given that patients with RH are known to be at an increased risk of developing more advanced cardiovascular conditions and complications, including HFrEF, it is important that early detection of RH occurs so that BP can be aggressively managed using a combination of pharmacological and non-pharmacological therapeutic strategies. This multi-disciplinary prevention approach for initiating treatment and maintaining the aggressive management of $\mathrm{BP}$ should be utilized irrespective of whether RH is diagnosed while a patient is still in the subclinical stage (A) of HF, or if RH is identified in a patient who has already progressed to HFrEF.

\section{Treatment Approaches}

Once a diagnosis of RH is established, treatment options should take into consideration the integrative approach of both pharmacological and non-pharmacological therapies. We provide in Fig. 1 a contemporary outline of available treatment strategies and recommended therapies for managing both $\mathrm{RH}$ and HFrEF. Although the efficacy of these collective treatment recommendations has not been tested in a randomized controlled clinical trial and confirmed specifically in patients with coexistent $\mathrm{RH}$ and HFrEF, the natural overlap of these upto-date interventions makes it logical to consider a combination of pharmacological and non-pharmacological therapies listed herein holds great promise for the effective long-term management of BP control in these individuals.

\section{Non-pharmacologic Interventions}

\section{Dietary Patterns and Sodium Intake}

The Dietary Approaches to Stop Hypertension (DASH) trial demonstrated that a diet consisting of a high proportion of fruits, vegetables, and low-fat dairy products as well as lower cholesterol and fat intake resulted in lower average BP measurements compared to an unrestricted diet [20]. However, when interpreting DASH trial results, it is noteworthy that the initial study did not employ a low-sodium diet and participants averaged a daily oral sodium intake of approximately $3000 \mathrm{mg}$. Subsequent follow-up studies focusing on sodium restriction in combination with the original DASH diet demonstrated an effective role of low sodium intake on BP reduction [21].

In patients with $\mathrm{RH}$, several recent studies confirm the benefits of sodium restriction on lowering BP. Despite a limited sample size, a study of 12 patients with RH showed that a low sodium diet $[50 \mathrm{mmol}$ per day, equivalent to approximately $1200 \mathrm{mg}$ ] correlated with a decrease in average office SBP/DBP measurements by $22.7 / 9.1 \mathrm{mmHg}$ as compared to a high-sodium diet $(250 \mathrm{mmol}$ per day, equivalent to approximately $5750 \mathrm{mg}$ ) [22]. While there remains some debate regarding the ideal daily sodium intake for patients with $\mathrm{RH}$, contemporary recommendations suggest reducing sodium intake to less than $2300 \mathrm{mg}$ daily is an achievable goal, with further reduction to less than $1500 \mathrm{mg}$ daily possibly reducing future morbidity and mortality $[4,6]$. Thus, because individual diet recommendations may vary based on patient needs and other comorbidities, in order to maximize potential dietary adherence, it is important to connect patients with nutrition support in order to ensure that personalized information is received.

Sodium restriction has classically been a component of HF management, with the ultimate goal of reducing fluid retention and optimizing fluid balance. However, there is still not a clear consensus on recommendations for sodium intake for patients with HF. While earlier ACC/AHA guidelines recommended sodium restriction in patients with symptomatic HF, subsequent 2013 guidelines downgraded the 


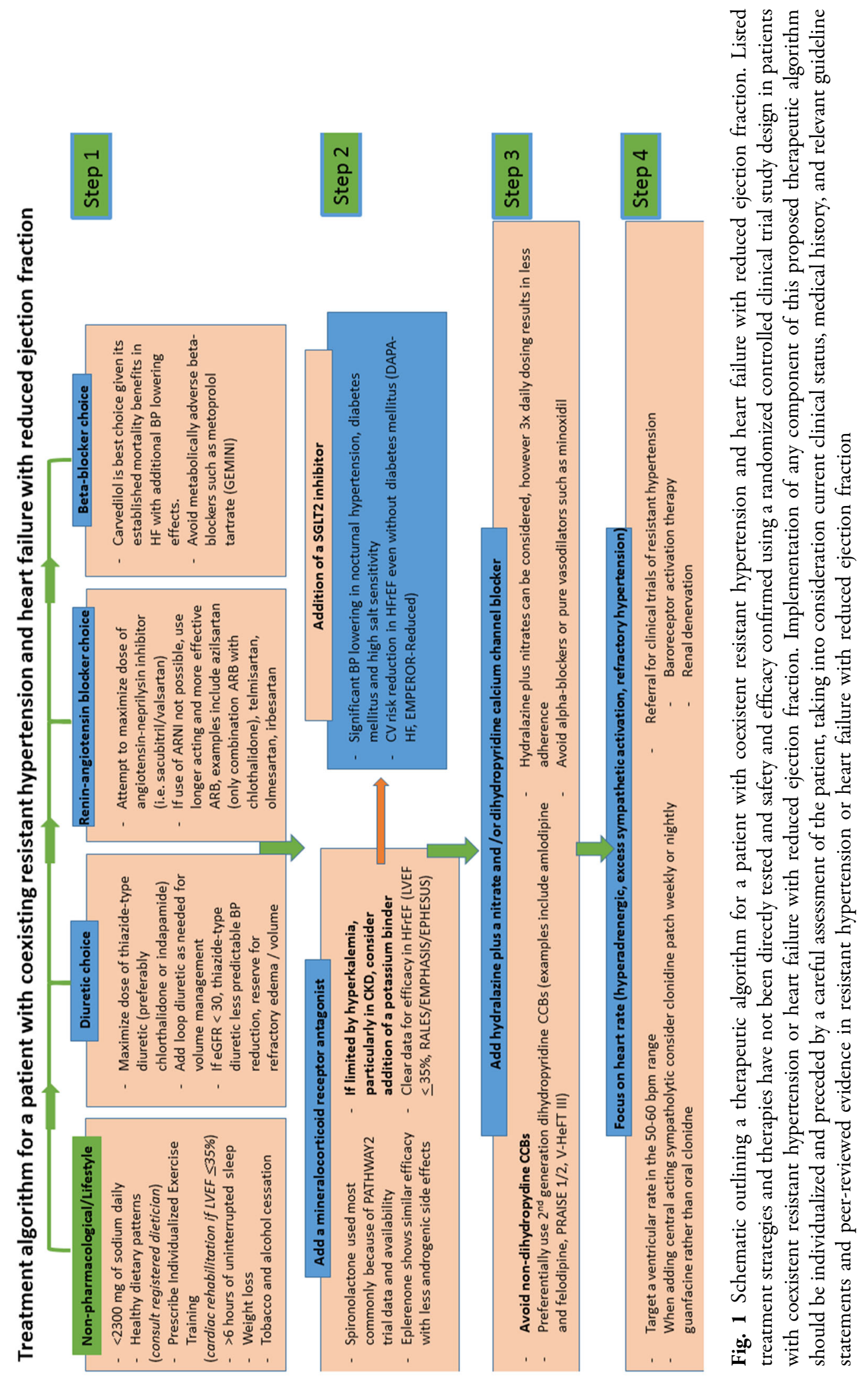


recommendation (Class IIa, level of evidence C) [23]. A wide range of evidence exists regarding sodium intake in $\mathrm{HF}$, with certain studies demonstrating benefits associated with sodium restriction while others demonstrate more positive outcomes with liberal sodium intake. A 2016 study examined data from the Heart Failure Adherence and Retention Trial and found that sodium intake of $<2500 \mathrm{mg}$ daily did not reduce the risk of death or hospitalization due to HF compared with a higher dietary intake of $>2500 \mathrm{mg}$ daily. The study did report a possible association between lower sodium intake and increased risk of HF hospitalizations, which contradicts past recommendations to limit dietary sodium intake for patients with HF [24]. While there currently remains no specific guideline for optimal daily sodium intake for patients with HFrEF, based on the collective body of evidence available, it is medically reasonable to recommend that patients with coexistent RH and HFrEF should adhere to a maximum daily dietary sodium intake of $2300 \mathrm{mg}$.

\section{Exercise and Physical Activity}

When exercise training is individually prescribed to patients as medicine, the contemporary view is that routine participation in structured moderate intensity aerobic exercise training can be an effective non-pharmacological intervention for both lowering and managing $\mathrm{BP}$ in patients with hypertension, including those treated for RH [4, 6, 25-27]. Indeed, it has been estimated that, on average, for patients with hypertension not specific to the RH subtype, extended participation in aerobic exercise training can account for up to an 8.3/5.2 $\mathrm{mmHg}$ drop in resting SBP/DBP [26], and an 3.2/ $2.7 \mathrm{mmHg}$ drop in daytime ambulatory SBP/ DBP [25]. For patients with $\mathrm{RH}$, the average therapeutic effect of aerobic exercise training on lowering daytime ambulatory SBP/DBP has also been reported to amount to at least $6 / 3 \mathrm{mmHg}$ [27], representing a two-fold-stronger therapeutic response for SBP as compared to that noted for patients with hypertension not identified by RH subtype [25].

The dose of exercise training needed to achieve a clinically relevant reduction in BP in those with $\mathrm{RH}$ is generally consistent with exercise dosing prescribed as necessary medical therapy for stable patients with HFrEF $[4,6,28-32]$. In the seminal randomized controlled clinical trial, Heart Failure: A Controlled Trial Investigating Outcomes of Exercise Training (HF-ACTION), patients who participated in at least 36 supervised exercise training sessions over 12 weeks demonstrated significantly larger improvements in exercise capacity and lower adjusted risk of both all-cause and cardiovascular related death as compared to counterparts randomized to usual care without exercise training [29]. The generalizable data reported in HF-ACTION and in related exercise training studies can be collectively taken to mean that it is medically reasonable for this form of nonpharmacological therapy to be discussed with stable patients with overlapping $\mathrm{RH}$ and HFrEF as a plan centered around aiming to accumulate at least $150 \mathrm{~min}$ of intentional moderate-intensity aerobic exercise spread out over the week; which would be further complemented by engaging in general physical activity promoting behaviors on all days of the week $[4,6,28,30-33]$. Whenever possible, it is also reasonable to highlight to select stable and exercise adherent patients that additional cardiovascular and musculoskeletal health benefits can be gained by incorporating into a training plan resistance exercise (1-3 sets of $12-15$ reps per large muscle group) performed on 2-3 nonconsecutive days per week [4, 6, 28-32].

Exercise training that is performed as medicine and based on individual capabilities and medical history can be expected for most stable patients with coexistent RH and HFrEF to be safe and medically appropriate. In clinically stable patients with moderate-to-severe HFrEF where the left ventricular ejection fraction percentage for some may be lower than $30 \%$, not only has the safety of exercise training been established, but patients who routinely participate in this therapy gain significant clinical benefits, including decreases in risk for both hospitalizations and death [29-32, 34-36].

The Centers for Medicare and Medicaid Services (CMS) recognizes the role of exercise training as medicine and considers this intervention a Class I (Level of Evidence: A) 
indicated therapy for clinically stable patients with HFrEF [29, 34, 35]. Accordingly, despite there being no large-scale clinical trial-based evidence available to confirm the role of exercise training as an effective medicine specifically for patients with coexistent RH and HFrEF, it should still be considered medically reasonable to prescribe for clinically stable individuals exercise training as a non-pharmacological therapy that has the strong likelihood of yielding improved clinical outcomes in addition to BP-lowering effects.

In contrast to what is known for the mechanistic associations between exercise and BP representative of the general population, there is a paucity of evidence and an incomplete mechanistic understanding of how exercise training yields beneficial effects on BP in those with $\mathrm{RH}$. This is a scientific area requiring directed efforts focusing on both mechanistic and patient-oriented clinical research, possibly centering on afferent pathways involving exercise-induced neuromuscular signaling changes. Examples of such phenomena have been frequently tested and observed in HFrEF [30, 31, 37-43]. In this context, many cases of refractory hypertension are suggested to be the result of excess and dysregulated sympathetic tone [6]. Therefore, with the possibility of an increase in parasympathetic tone acting to counteract exaggerated sympathoexcitation, it is conceivable that if such re-balancing of neural signaling stemming from muscle afferents can occur as a result of exercise training consistent with what has been observed in HFrEF alone [43], benefits gained by overlap patients may include for example, lower resting heart rates, a more proportional rise in heart rate relative to work-rate, lower circulating norepinephrine levels, and baroreflex sensitivity resetting [44-48]. These and other exercise training induced adaptations when coupled with improvements in functional capacity should be expected to contribute to less severe clinical status in patients exhibiting the RH and HFrEF overlap.

Given that it is apparent that even in a highrisk patient group, such as HFrEF, regular participation in moderate-intensity exercise training can typically be safe and results in improved physiological function and clinical status, multi-disciplinary interventions aimed at achieving optimal decreases in BP and sustained control at such goal levels in clinically stable patients with coexistent RH and HFrEF should consider it necessary to include in any prevention plan exercise training therapy that largely mirrors the proven exercise-based cardiac rehab model established for HFrEF [29-32, 34-36].

\section{Pharmacological Interventions}

\section{Renin-Angiotensin-Aldosterone Blockers with Neprilysin Inhibition}

Drugs that target the RAAS system are crucial for the management of BP in hypertensive patients as well as typical signs and symptoms related to HFrEF. In patients with primary hypertension, the use of angiotensin-converting enzyme inhibitors (ACE-I) and/or angiotensin receptor blockers (ARB) are often employed as first-line therapies for effectively managing BP $[4,49]$. In patients with HFrEF, the efficacy of ACE-I/ARB when used alongside other guideline therapies has also been consistently supported [35].

However, there are select ambulatory patients with HFrEF who remain symptomatic despite being optimally treated with the combination of an ACE-I/ARB, beta-blocker, and MRA, where guidelines now recommend based on contemporary evidence that better clinical outcomes are likely when ACE-I/ARB are replaced with an angiotensin receptor-neprilysin inhibitor (ARNI) (i.e., sacubitril/valsartan) [8,50-55]. While a similar level of evidence supporting the use of an ARNI to specifically treat $\mathrm{RH}$ is not available [56], a limited number of data are available to highlight that this therapy can yield clear BP reductions in select patients with primary hypertension [57]. For example, a 2010 study of 1328 patients aged 18-75 years with mild-tomoderate hypertension demonstrated that sacubitril/valsartan compared with valsartan alone resulted in greater BP lowering and similar tolerability [58]. Thus, although randomized controlled clinical trial data on the efficacy and safety of using an ARNI in patients with RH is not available, the totality of data in HFrEF 
coupled with expert opinion can be taken to suggest that in select ambulatory patients with concomitant RH and HFrEF, use of an ARNI should be considered a possible first-line therapy after taking into consideration the known contraindications to its use in HFrEF $[8,50,51,57,58]$.

\section{Beta-blockers}

When managing primary hypertension alone, beta-blockers may be used in combination with other medication classes, but are no longer considered a first-line therapy unless compelling reasons are present indicating otherwise [4]. HFrEF is one example of an indication where the use of beta-blockers has been shown to reduce the effects of dysregulated sympathetic activation, slow or prevent progressive pathologic remodeling, and lessen the risk of cardiovascular-related mortality. Thus, in contemporary HF guidelines, use of beta-blocker therapy is strongly recommended [23].

Concomitant management of $\mathrm{RH}$ and HFrEF requires choosing a beta-blocker that will best control BP but also has randomized control trial data demonstrating a mortality benefit in HFrEF. High-quality data for mortality reduction in HFrEF are seen with bisoprolol, carvedilol, and metoprolol succinate [23]. Use of vasodilating beta-blockers such as carvedilol not only lower BP to a greater extent, but have neutral effects on risk factors for subsequent cardiovascular disease, such as blood glucose [59].

\section{Diuretics}

Sub-optimal volume status is posited to underlie many cases of $\mathrm{RH}$, therefore diuretics that are long-acting and appropriately dosed for level of kidney function remain an important component of $\mathrm{RH}$ management [6]. Thiazide-type diuretics, including chlorthalidone and indapamide, are most effective in lowering BP in patients with RH. Chlorthalidone demonstrates superiority to hydrochlorothiazide at comparable doses and shows effective BP lowering among patients with an eGFR greater than $30 \mathrm{ml} / \mathrm{min} / 1.73 \mathrm{~m}^{2}$ [60-63]. Thiazide-type diuretics are not as effective for BP lowering below this eGFR threshold, and use of loop diuretics, such as furosemide, torsemide, or bumetanide, should be considered. Thiazidetype diuretics can be stopped in this setting and substituted with a loop diuretic, or alternatively sequential nephron blockade can be achieved with both taken simultaneously, which has been proven effective in CKD patients with $\mathrm{RH}$ [64].

Diuretics are also used in HF management in order to optimize fluid status. In contrast to $\mathrm{RH}$, loop diuretics are often the preferred diuretic therapy in HF management because of the more pronounced, albeit shorter-acting, diuretic effects [65]. The most recent HF guidelines recommend diuretics for all patients with HFrEF who demonstrate evidence of fluid overload in order to reduce HF-related symptoms [23].

In the setting of coexistent RH and HFrEF, efforts should be made to add or substitute a thiazide-type diuretic, which will result in more effective BP lowering than a loop diuretic alone. Because such a substitution may result in worsening congestion, close monitoring of a patient's renal function and volume status is critical. At an eGFR of less than $30 \mathrm{ml} / \mathrm{min} /$ $1.73 \mathrm{~m}^{2}$, use of thiazide-type diuretics is generally reserved for refractory edema/volume, and does not provide predictable BP lowering.

\section{Mineralocorticoid Receptor Antagonists}

Elevated aldosterone that is independent from RAAS control is a common cause of hypertension and occurs in approximately $7-20 \%$ of patients with RH $[6,66]$. Guidelines report that on top of the use of diuretics, MRAs are now among the drug of choice for the treatment of $\mathrm{RH}$ [6]. Strong support for this recommendation comes from evidence generated from the PATHWAY-2 trial, which involved the study of patients with $\mathrm{RH}$ previously managed on a three-drug regimen including a RAAS blocker, $\mathrm{CCB}$, and a diuretic, to which a fourth medication was added (spironolactone, bisoprolol, doxazosin, or placebo) [67]. Key data reported from PATHWAY-2 clearly demonstrated that spironolactone lowered BP more effectively than placebo, bisoprolol, or doxazosin [67].

A substudy of PATHWAY-2 assessed if amiloride could be used as an alternative to 
spironolactone. This study was an open-label extension of the main PATHWAY-2 trial in participants who were willing to crossover from spironolactone to amiloride for 6 to 12 weeks. Amiloride at doses between 10 and $20 \mathrm{mg}$ daily reduced home systolic BP by $20 \mathrm{mmHg}$, which was comparable to reductions seen in the primary trial with spironolactone [68].

MRAs are also recommended for patients with HFrEF to reduce symptoms of HF as well as associated mortality. Key data supporting the efficacy of MRAs in the management of HF comes from the Randomized Aldactone Evaluation Study (RALES) where spironolactone was observed to lower morality risk by approximately 30\% in patients with HFrEF [69]. Additional studies of eplerenone in HFrEF, including the EPHESUS and EMPHASIS, demonstrate that eplerenone is also effective for decreasing the risk of HF-related hospitalizations and cardiovascular mortality [70, 71].

Given the evidence supporting the efficacy of MRAs in treating both HFrEF and RH, medications falling within this class of therapy should be prescribed regularly in patients with coexistent RH and HFrEF. However, it is noteworthy that for select patients where MRAs are prescribed close monitoring of renal function and electrolyte levels is necessary since use of this therapy is traditionally contraindicated in the presence of elevated serum creatinine and/or hyperkalemia. Use of potassium binders (discussed below) may obviate the risk of hyperkalemia.

\section{Sodium-Glucose Cotransporter-2 Inhibitors}

Sodium-glucose cotransporter-2 (SGLT2) inhibitors, which include empagliflozin, canagliflozin, and dapagliflozin, block reabsorption of glucose in the proximal tubule of the kidney, resulting in substantial glycosuria. SGLT2 inhibitors were initially developed for the management of type 2 diabetes mellitus. However, recent data support the use of these medications in HFrEF patients without diabetes. Both empagliflozin and dapagliflozin have been reported to lower the risk of cardiovascular death or hospitalization for HF when compared with placebo, regardless of the presence or absence of diabetes [72, 73].
In addition to the demonstrated benefits regarding blood sugar control and reduction of HF events, SGLT2 inhibitors can also lead to reductions in BP. While the exact mechanism responsible for lowering BP is not clear, it is likely a combination of osmotic diuresis from glycosuria and natriuresis, which causes an average reduction of $3.6 / 1.7 \mathrm{mmHg}$ in $24-\mathrm{h}$ ambulatory SBP/DBP. Such a reduction is comparable to the effect of a low-dose thiazide-like diuretic $[74,75]$. The BP-lowering role of SGLT2 inhibitors extends to special populations. A randomized trial involving elderly diabetic patients demonstrated improved nocturnal hypertension control with empagliflozin [76]. Given the multiple beneficial effects of SGLT2 inhibitors, they should be included in the treatment regimen for $\mathrm{RH}$ and co-existing HFrEF, particularly if the BP remains uncontrolled after addition of an MRA.

\section{Direct Vasodilators and Calcium Channel Blockers}

Hydralazine is a direct-acting vasodilator and has been used for the treatment of hypertension since the 1950s. Hydralazine modestly lowers BP but has generally been supplanted by newer antihypertensive therapies that are better tolerated and are taken once daily [77].

Early HF trials of hydralazine in combination with nitrate therapy demonstrate a mortality benefit in select patients with HFrEF, but it must be noted that these trials did not take place in the setting of contemporary HF therapy $[78,79]$. In the V-HeFT I trial, no patients were taking a beta-blocker or ACE-inhibitor. Hydralazine plus isosorbide dinitrate reduced all-cause mortality at 2 years as compared to placebo. In the V-HeFT II trial, no patients were treated with beta-blockers, and participants were randomized to enalapril or hydralazine plus isosorbide dinitrate. Participants taking enalapril demonstrated a lower mortality rate at 2 years. Given the above data, the use of hydralazine in combination with a nitrate can be considered when treating patients with $\mathrm{RH}$ and HFrEF. This combination should only be added after maximizing doses of ARNI, betablocker, MRA, diuretic, and SGLT2-inhibitor. How much the combination of hydralazine and 
nitrate impacts adverse cardiovascular events in the background of contemporary HF therapy is unknown? A major limiting factor in the use of hydralazine and a nitrate is the need for multiple doses per day and may greatly limit patient adherence.

Dihydropyridine CCBs are among first-line medications for the treatment of primary hypertension [4]. However, the role of these medications in patients with hypertension and coexistent HFrEF is less prominent. Unlike betablockers and blockers of the RAAS, there is no mortality benefit seen in studies of dihydropyridine CCBs in HFrEF. Second-generation dihydropyridine CCBs such as amlodipine and felodipine have minimal to no negative inotropic activity when prescribed at usual doses and are safe to use to treat hypertension in HFrEF. However, the use of these medications does not result in improved patient survival [80, 81]. Non-dihydropyridine CCBs such as verapamil and diltiazem should generally be avoided because they do not provide any cardiovascular risk reduction for patients with HFrEF, and may worsen outcomes [82]. Given the desire to maximize doses of ARNIs, betablockers, mineralocorticoid receptor antagonists in HFrEF, dihydropyridine CCBs should be used sparingly, and generally as a fifth or sixth agent to treat hypertension.

\section{Potassium Binders}

Hyperkalemia is associated with an increased risk of adverse events, including cardiac arrhythmias. Many medications that treat both HFrEF and RH increase serum potassium, including ARNIs and MRAs. Additionally, many patients with both HFrEF and $\mathrm{RH}$ have CKD, which further disrupts potassium homeostasis. Elevated serum potassium levels may preclude concomitant use of these medication classes or may result in withdrawal of these effective medications [83]. Thus, strategies to maintain normal serum potassium levels are needed.

Use of loop and thiazide diuretics, in combination with a low-potassium diet, should be the first approach undertaken to lower serum potassium levels before considering adding medications specifically for potassium lowering. Historical treatment for chronic hyperkalemia includes sodium polystyrene sulfonate, a cation-exchange resin that binds potassium in the colon. However, the modern use of this treatment approach has fallen out of favor and is no longer recommended given the lack of efficacy data and the presence of an association with colonic necrosis.

Relatively new therapies include patiromer, a cation-exchange polymer that binds potassium in the colon, and zirconium cyclosilicate, which exchanges both sodium and hydrogen ions for potassium while it transits the gastrointestinal tract. Zirconium cyclosilicate has proven effective in potassium lowering in patients with $\mathrm{HF}$ and multiple cardiovascular comorbidities including CKD and diabetes mellitus [84, 85]. The pivotal study of patiromer enrolled 243 patients with CKD who were receiving RAAS blockers (42\% had concomitant HF and 97\% had hypertension) use of patiromer was associated with a decrease in serum potassium levels and, when compared with placebo, a reduction in the recurrence of hyperkalemia [86].

Patients with RH and HFrEF are at risk of complications associated with hyperkalemia, but also stand to benefit from medication classes that increase serum potassium. Treatment with newer potassium binding agents should be strongly considered if needed to optimize potassium levels and better control BP.

\section{Future Treatment Options}

The management of coexisting $\mathrm{RH}$ and HFrEF becomes complex for physicians and patients when considering the numerous drugs and treatment combinations available. While patients may trial several medication combinations in order to identify the most effective regimen, treatment options beyond currently available pharmacotherapies are currently under investigation. The below therapies may be incorporated into future practice if ongoing clinical trials demonstrate favorable outcomes.

\section{Small Interfering RNAs}

Small interfering RNAs (siRNAs) that target angiotensinogen production in the liver, with the ultimate goal of limiting RAAS activity, 
represent a potential future management option for RH. SiRNAs are given as subcutaneous injections, administered approximately every 4 weeks. Studies on animal models demonstrate significant reductions in BP with the use of targeted siRNAs [87, 88]. Phase 1 human trials are currently ongoing (clinicaltrials.gov identifier: NCT03934307), with phase 2 trials still in planning stages. SiRNAs, for example inclisiran, have already demonstrated efficacy for the management of dyslipidemia in the setting of atherosclerotic cardiovascular disease [89]. These are an exciting new class of medications that may prove beneficial in the treatment of $\mathrm{RH}$.

\section{Renal Denervation}

The evidence in support of the use of renal denervation in the treatment of $\mathrm{RH}$ is varied [90]. Multiple unblinded studies suggest renal denervation can result in lowered BP; although, the pivotal SYMPLICITY-HTN 3 trial in patients with $\mathrm{RH}$ and did not demonstrate a significant $\mathrm{BP}$ reduction difference between the intervention arm and sham control arm [91]. Other smaller trials of different catheter-based technologies have shown the possibility for limited BP lowering effects yielded by such therapy $[92,93]$. A study of the SPYRAL renal denervation catheter in patients not taking BP-lowering medications demonstrated a statistically significant decrease in BP as measured by $24-\mathrm{h}$ ABPM [94]; although to what extent renal denervation lowers BP in patients with RH is still unknown. Substudy data from the SYMPLICITY-HTN 3 trial are still being scrutinized, analyzed, and interpreted for an improved understanding of reasons for primary outcome results, and ongoing trials of renal denervation for $\mathrm{RH}$ include the SPYRAL-ON trial (clinicaltrials.gov identifier: NCT02439775) and the TARGET BP 1 trial (clinicaltrials.gov identifier: NCT02910414).

Given that a hallmark feature of HFrEF is dysregulated sympathetic activity, resulting in chronically elevated sympathetic activation, renal denervation may eventually emerge as an effective treatment tool for this population. A 2017 systematic review and meta-analysis of renal denervation in patients with HFrEF suggests that in addition to possible BP lowering effects, this advanced therapy may also yield improvements in LV systolic function, amongst other physiological adaptations [95]. Thus, patients with RH and HFrEF may derive substantiative clinical benefits from renal denervation therapy, although this is a hypothesis that has not been supported with data generated from a dedicated randomized controlled clinical trial.

\section{Baroreflex Activation Therapy}

Baroreflex activation therapy (BAT) has been studied in the treatment of both RH and HFrEF. Recently, the Rheos Pivotal Trial enrolled 265 patients with $\mathrm{RH}$ who underwent surgical implantation with a device designed to stimulate the carotid baroreceptors. Although participants achieved lowered BP, the study failed to meet two of five primary endpoints, including a safety end-point, and thus did not receive approval by the US Food and Drug Administration to treat RH [96]. Therefore, while BAT has the potential to yield decreased BP in patients with $\mathrm{RH}$, this treatment is not currently approved for medical use for this indication in the US.

A less invasive and newer iteration of the implant previously studied and discontinued in hypertension, the so-called BAROSTIM NEO system (CVRx, Minneapolis, MN, USA) has been developed for study in HFrEF. Using a multicenter and randomized controlled trial study design, the Baroreflex Activation Therapy for Heart Failure trial prospectively enrolled and randomized individuals with HFrEF (LVEF < $35 \%$ ) in a $1: 1$ ratio to receive either BAT using the BAROSTIM NEO system plus optimal medical management or optimal medical management alone [97]. The key results published in 2020 not only confirmed the safety of BAT, but participants randomized to this arm also demonstrated significant baseline to follow up improvements in quality of life, exercise capacity, and NT-proBNP; changes which were all significantly greater than those observed for control arm patients [97].

A newer but similar technology that is inserted into the carotid sinus by endovascular deployment, rather a surgical implant, is 
currently enrolling in a pivotal clinical trial for the treatment of RH. Initial study of this therapy occurred in the CALM first-in-man trial, demonstrating in 30 participants that this nextgeneration therapy for lowering BP is both safe and effective [98]. The pivotal CALM-2 trial is currently enrolling patients (clinicaltrials.gov identifier: NCT03179800).

\section{CONCLUSIONS}

As the number of patients with $\mathrm{RH}$ continues to rise in the US, so too does the prevalence of patients with HFrEF and also those with coexistent RH and HFrEF. While more directed research is needed to advance the mechanistic understanding of associations between $\mathrm{BP}, \mathrm{RH}$, HFrEF, and adverse clinical outcomes, patients diagnosed with both $\mathrm{RH}$ and $\mathrm{HFrEF}$ require treatment for high BP right now. Both pharmacological and non-pharmacological therapies are currently available and can be used to effectively target and aggressively manage BP control in patients with coexistent $\mathrm{RH}$ and HFrEF. As more higher-quality data become available as a result of cutting-edge therapeutic trials, it can be expected that the continued evolution of BP treatment guideline recommendations will ultimately allow for the advanced development of individualized treatment plans that can durably maintain BP below guideline-recommended targets.

\section{ACKNOWLEDGEMENTS}

Funding. No funding or sponsorship was received for this study or publication of this article.

Authorship. All named authors meet the International Committee of Medical Journal Editors (ICMJE) criteria for authorship for this article, take responsibility for the integrity of the work as a whole, and have given their approval for this version to be published.
Disclosures. Luke J. Laffin is a Vascular Dynamics-Hypertension Committee member and LucidAct Health-Medical Advisor. Katherine Lang and Erik $\mathrm{H}$. Van Iterson have nothing to disclose.

Compliance with ethics guidelines. This article is based on previously conducted studies and does not contain any new studies with human participants or animals performed by any of the authors.

Data availability. Data sharing is not applicable to this article as no datasets were generated or analyzed during the current study.

Open Access. This article is licensed under a Creative Commons Attribution-NonCommercial 4.0 International License, which permits any non-commercial use, sharing, adaptation, distribution and reproduction in any medium or format, as long as you give appropriate credit to the original author(s) and the source, provide a link to the Creative Commons licence, and indicate if changes were made. The images or other third party material in this article are included in the article's Creative Commons licence, unless indicated otherwise in a credit line to the material. If material is not included in the article's Creative Commons licence and your intended use is not permitted by statutory regulation or exceeds the permitted use, you will need to obtain permission directly from the copyright holder. To view a copy of this licence, visit http://creativecommons.org/licenses/bync/4.0/.

\section{REFERENCES}

1. Lawes CM, Vander Hoorn S, Rodgers A, International Society of H. Global burden of blood-pressure-related disease, 2001. Lancet. 2008;371: 1513-8.

2. Collaboration NCDRF. Worldwide trends in blood pressure from 1975 to 2015: a pooled analysis of 1479 population-based measurement studies with 191 million participants. Lancet. 2017;389:37-55. 
3. Sprint Research Group, Wright JT Jr, Williamson JD, et al. A randomized trial of intensive versus standard blood-pressure control. $\mathrm{N}$ Engl J Med. 2015;373:2103-16.

4. Whelton PK, Carey RM, Aronow WS, et al. ACC/ AHA/AAPA/ABC/ACPM/AGS/APhA/ASH/ASPC/ NMA/PCNA Guideline for the Prevention, Detection, Evaluation, and Management of High Blood Pressure in Adults: a Report of the American College of Cardiology/American Heart Association Task Force on Clinical Practice Guidelines. J Am Coll Cardiol. 2017;71:e127-248.

5. Virani SS, Alonso A, Benjamin EJ, et al. Heart disease and stroke statistics-2020 update: a report from the American Heart Association. Circulation. 2020;141:e139-596.

6. Carey RM, Calhoun DA, Bakris GL, et al. Resistant hypertension: detection, evaluation, and management: a scientific statement from the American Heart Association. Hypertension. 2018;72:e53-90.

7. Dudenbostel T, Siddiqui M, Gharpure N, Calhoun DA. Refractory versus resistant hypertension: novel distinctive phenotypes. J Nat Sci 2017;3(9).

8. Yancy CW, Jessup M, Bozkurt B, et al. 2017 ACC/ AHA/HFSA focused update of the 2013 ACCF/AHA guideline for the management of heart failure: a report of the American College of Cardiology/ American Heart Association Task Force on Clinical Practice Guidelines and the Heart Failure Society of America. Circulation. 2017;136:e137-61.

9. Breslow NE, Day NE (1987) Effects of treatment on morbidity in hypertension II: results in patients with diastolic blood pressure averaging 90 through 114 mmHg. JAMA 131-5.

10. Kostis JB, Davis BR, Cutler J, et al. Prevention of heart failure by antihypertensive drug treatment in older persons with isolated systolic hypertension. SHEP Cooperative Research Group. JAMA. 1997;278:212-6.

11. Egan BM, Zhao Y, Axon RN, Brzezinski WA, Ferdinand $\mathrm{KC}$. Uncontrolled and apparent treatment resistant hypertension in the United States, 1988 to 2008. Circulation. 2011;124:1046-58.

12. Pimenta E, Calhoun DA. Resistant hypertension: incidence, prevalence, and prognosis. Circulation. 2012;125:1594-6.

13. Patel KV, Li X, Kondamudi N, et al. Prevalence of apparent treatment-resistant hypertension in the United States according to the 2017 high blood pressure guideline. Mayo Clin Proc. 2019;94: 776-82.
14. United States Census Bureau. Table 2 Projected age and sex composition of the population. Projections for the United States. Suitland: United States Census Bureau; 2017. p. 2017-60.

15. Levy D, Kenchaiah S, Larson MG, et al. Long-term trends in the incidence of and survival with heart failure. N Engl J Med. 2002;347:1397-402.

16. Jackson SL, Tong X, King RJ, Loustalot F, Hong Y, Ritchey MD. National burden of heart failure events in the United States, 2006 to 2014. Circ Heart Fail. 2018;11:e004873.

17. Jin CN, Liu M, Sun JP, et al. The prevalence and prognosis of resistant hypertension in patients with heart failure. PLoS ONE. 2014;9:e114958.

18. Daugherty SL, Powers JD, Magid DJ, et al. Incidence and prognosis of resistant hypertension in hypertensive patients. Circulation. 2012;125:1635-42.

19. Sim JJ, Bhandari SK, Shi J, et al. Comparative risk of renal, cardiovascular, and mortality outcomes in controlled, uncontrolled resistant, and nonresistant hypertension. Kidney Int. 2015;88:622-32.

20. Appel LJ, Moore TJ, Obarzanek E, et al. A clinical trial of the effects of dietary patterns on blood pressure. DASH Collaborative Research Group. N Engl J Med. 1997;336:1117-24.

21. Sacks FM, Svetkey LP, Vollmer WM, et al. Effects on blood pressure of reduced dietary sodium and the Dietary Approaches to Stop Hypertension (DASH) diet. DASH-Sodium Collaborative Research Group. N Engl J Med. 2001;344:3-10.

22. Pimenta E, Gaddam KK, Oparil S, et al. Effects of dietary sodium reduction on blood pressure in subjects with resistant hypertension: results from a randomized trial. Hypertension. 2009;54:475-81.

23. Writing Committee M, Yancy CW, Jessup M, et al. 2013 ACCF/AHA guideline for the management of heart failure: a report of the American College of Cardiology Foundation/American Heart Association Task Force on practice guidelines. Circulation. 2013;128:e240-327.

24. Doukky R, Avery E, Mangla A, et al. Impact of dietary sodium restriction on heart failure outcomes. JACC Heart Fail. 2016;4:24-35.

25. Cornelissen VA, Buys R, Smart NA. Endurance exercise beneficially affects ambulatory blood pressure: a systematic review and meta-analysis. J Hypertens. 2013;31:639-48.

26. Cornelissen VA, Smart NA. Exercise training for blood pressure: a systematic review and meta-analysis. J Am Heart Assoc. 2013;2:e004473. 
27. Dimeo F, Pagonas N, Seibert F, Arndt R, Zidek W, Westhoff TH. Aerobic exercise reduces blood pressure in resistant hypertension. Hypertension. 2012;60:653-8.

28. Piercy KL, Troiano RP, Ballard RM, et al. The physical activity guidelines for Americans. JAMA. 2018;320:2020-8.

29. O'Connor CM, Whellan DJ, Lee KL, et al. Efficacy and safety of exercise training in patients with chronic heart failure: HF-ACTION randomized controlled trial. JAMA. 2009;301:1439-50.

30. Taylor RS, Sagar VA, Davies EJ, et al. Exercise-based rehabilitation for heart failure. Cochrane Database Syst Rev. 2014;2014:CD003331.

31. Taylor RS, Walker S, Smart NA, et al. Impact of exercise-based cardiac rehabilitation in patients with heart failure (ExTraMATCH II) on mortality and hospitalisation: an individual patient data meta-analysis of randomised trials. Eur J Heart Fail. 2018;20:1735-43.

32. Piepoli MF, Corra U, Benzer W, et al. Secondary prevention through cardiac rehabilitation: from knowledge to implementation. A position paper from the Cardiac Rehabilitation Section of the European Association of Cardiovascular Prevention and Rehabilitation. Eur J Cardiovasc Prev Rehabil. 2010;17:1-17.

33. Fletcher GF, Ades PA, Kligfield P, et al. Exercise standards for testing and training: a scientific statement from the American Heart Association. Circulation. 2013;128:873-934.

34. Decision Memorandum for Coverage of Cardiac Rehabilitation (CR) Programs for Chronic Heart Failure (HF). The Centers for Medicare \& Medicaid Services (CMS). § 41049(b)(1)(vii). United States of America, 2014:24.

35. Yancy C, Jessup M, Bozkurt B, et al. ACCF/AHA guideline for the management of heart failure: a report of the American College of Cardiology Foundation/American Heart Association Task Force on Practice Guidelines. Circulation. 2013;128: $1810-52$.

36. Austin J, Williams WR, Ross L, Hutchison S. Fiveyear follow-up findings from a randomized controlled trial of cardiac rehabilitation for heart failure. Eur J Cardiovasc Prev Rehabil. 2008;15:162-7.

37. Van Iterson EH, Johnson BD, Joyner MJ, Curry TB, Olson TP. Vo2 kinetics associated with moderateintensity exercise in heart failure: impact of intrathecal fentanyl inhibition of group III/IV locomotor muscle afferents. Am J Physiol Heart Circ Physiol. 2017;313:H114-24.
38. Van Iterson EH, Snyder EM, Joyner MJ, Johnson BD, Olson TP. Intrathecal fentanyl blockade of afferent neural feedback from skeletal muscle during exercise in heart failure patients: Influence on circulatory power and pulmonary vascular capacitance. Int J Cardiol. 2015;201:384-93.

39. Van Iterson EH, Snyder EM, Johnson BD, Olson TP. Influence of the metaboreflex on pulmonary vascular capacitance in heart failure. Med Sci Sports Exerc. 2016;48:353-62.

40. SmithJR Joyner MJ, Curry TB, Borlaug BA, KellerRoss ML, Van Iterson EH, Olson TP. Locomotor muscle group III/IV afferents constrain stroke volume and contribute to exercise intolerance in human heart failure. J Physiol. 2020; https://doi. org/10.1113/JP280333.

41. Coats AJS, Forman DE, Haykowsky M, et al. Physical function and exercise training in older patients with heart failure. Nat Rev Cardiol. 2017;14:550-9.

42. Piepoli M, Ponikowski P, Clark AL, Banasiak W, Capucci A, Coats AJS. A neural link to explain the "muscle hypothesis" of exercise intolerance in chronic heart failure. Am Heart J. 1999;137:1050-6.

43. Piepoli M, Clark AL, Volterrani M, Adamopoulos S, Sleight P, Coats AJ. Contribution of muscle afferents to the hemodynamic, autonomic, and ventilatory responses to exercise in patients with chronic heart failure: effects of physical training. Circulation. 1996;93:940-52.

44. Blomqvist CG, Saltin B. Cardiovascular adaptations to physical training. Annu Rev Physiol. 1983;45: 169-89.

45. Goldsmith RL, Bigger JT Jr, Steinman RC, Fleiss JL. Comparison of 24-hour parasympathetic activity in endurance-trained and untrained young men. J Am Coll Cardiol. 1992;20:552-8.

46. Hartley LH, Mason JW, Hogan RP, et al. Multiple hormonal responses to prolonged exercise in relation to physical training. J Appl Physiol. 1972;33: 607-10.

47. La Rovere MT, Bersano C, Gnemmi M, Specchia G, Schwartz PJ. Exercise-induced increase in baroreflex sensitivity predicts improved prognosis after myocardial infarction. Circulation. 2002;106: 945-9.

48. Joyner MJ. Baroreceptor function during exercise: resetting the record. Exp Physiol. 2006;91:27-36.

49. Unger T, Borghi C, Charchar F, et al. 2020 international society of hypertension global hypertension practice guidelines. Hypertension. 2020;75: 1334-57. 
50. McMurray JJ, Packer M, Desai AS, et al. Angiotensinneprilysin inhibition versus enalapril in heart failure. N Engl J Med. 2014;371:993-1004.

51. Seferovic PM, Ponikowski P, Anker SD, et al. Clinical practice update on heart failure 2019: pharmacotherapy, procedures, devices and patient management. An expert consensus meeting report of the Heart Failure Association of the European Society of Cardiology. Eur J Heart Fail. 2019;21: 1169-86.

52. Jhund PS, Fu M, Bayram E, et al. Efficacy and safety of LCZ696 (sacubitril-valsartan) according to age: insights from PARADIGM-HF. Eur Heart J. 2015;36: 2576-84.

53. Simpson J, Jhund PS, Cardoso JS, et al. Comparing LCZ696 with enalapril according to baseline risk using the MAGGIC and EMPHASIS-HF risk scores: an analysis of mortality and morbidity in PARADIGM-HF. J Am Coll Cardiol. 2015;66:2059-71.

54. Okumura N, Jhund PS, Gong J, et al. Effects of Sacubitril/Valsartan in the PARADIGM-HF trial (prospective comparison of ARNI with ACEI to determine impact on global mortality and morbidity in heart failure) according to background therapy. Circ Heart Fail. 2016;9:e003212.

55. Velazquez EJ, Morrow DA, DeVore $\mathrm{AD}$, et al. Angiotensin-neprilysin inhibition in acute decompensated heart failure. N Engl J Med. 2019;380: 539-48.

56. Kjeldsen SE, Narkiewicz K, Burnier M, Oparil S. Will we ever use angiotensin receptor neprilysin inhibition (ARNi) for the treatment of hypertension? Blood Press. 2019;28:75-6.

57. Anderson SL, Marrs JC. Sacubitril/valsartan: evaluation of safety and efficacy as an antihypertensive treatment. Drugs Context. 2018;7:212542.

58. Ruilope LM, Dukat A, Bohm M, Lacourciere Y, Gong J, Lefkowitz MP. Blood-pressure reduction with LCZ696, a novel dual-acting inhibitor of the angiotensin II receptor and neprilysin: a randomised, double-blind, placebo-controlled, active comparator study. Lancet. 2010;375:1255-66.

59. Bakris GL, Fonseca V, Katholi RE, et al. Metabolic effects of carvedilol vs metoprolol in patients with type 2 diabetes mellitus and hypertension: a randomized controlled trial. JAMA. 2004;292:2227-36.

60. Reubi FC, Cottier PT. Effects of reduced glomerular filtration rate on responsiveness to chlorothiazide and mercurial diuretics. Circulation. 1961;23: 200-10.
61. DiNicolantonio JJ, Bhutani J, Lavie CJ, O'Keefe JH. Evidence-based diuretics: focus on chlorthalidone and indapamide. Future Cardiol. 2015;11:203-17.

62. Kaplan NM. The choice of thiazide diuretics: why chlorthalidone may replace hydrochlorothiazide. Hypertension. 2009;54:951-3.

63. Roush GC, Sica DA. Diuretics for hypertension: a review and update. Am J Hypertens. 2016;29: 1130-7.

64. Bobrie G, Frank M, Azizi M, et al. Sequential nephron blockade versus sequential renin-angiotensin system blockade in resistant hypertension: a prospective, randomized, open blinded endpoint study. J Hypertens. 2012;30:1656-64.

65. Casu G, Merella P. Diuretic therapy in heart failure-current approaches. Eur Cardiol. 2015;10: 42-7.

66. Brown JM, Siddiqui M, Calhoun DA, et al. The unrecognized prevalence of primary aldosteronism: a cross-sectional study. Ann Intern Med. 2020;173: 10-20.

67. Williams B, MacDonald TM, Morant S, et al. Spironolactone versus placebo, bisoprolol, and doxazosin to determine the optimal treatment for drug-resistant hypertension (PATHWAY-2): a randomised, double-blind, crossover trial. Lancet. 2015;386:2059-68.

68. Williams B, MacDonald TM, Morant SV, et al. Endocrine and haemodynamic changes in resistant hypertension, and blood pressure responses to spironolactone or amiloride: the PATHWAY-2 mechanisms substudies. Lancet Diabetes Endocrinol. 2018;6:464-75.

69. Pitt B, Zannad F, Remme WJ, et al. The effect of spironolactone on morbidity and mortality in patients with severe heart failure. Randomized Aldactone Evaluation Study Investigators. N Engl J Med. 1999;341:709-17.

70. Zannad F, McMurray JJ, Krum H, et al. Eplerenone in patients with systolic heart failure and mild symptoms. N Engl J Med. 2011;364:11-21.

71. Pitt B, Remme W, Zannad F, et al. Eplerenone, a selective aldosterone blocker, in patients with left ventricular dysfunction after myocardial infarction. N Engl J Med. 2003;348:1309-21.

72. McMurray JJV, Solomon SD, Inzucchi SE, et al. Dapagliflozin in patients with heart failure and reduced ejection fraction. N Engl J Med. 2019;381: 1995-2008. 
73. Packer M, Anker SD, Butler J, et al. Cardiovascular and renal outcomes with empagliflozin in heart failure. N Engl J Med. 2020;383:1413-24.

74. Briasoulis A, Al Dhaybi O, Bakris GL. SGLT2 inhibitors and mechanisms of hypertension. Curr Cardiol Rep. 2018;20:1.

75. Georgianos PI, Agarwal R. Ambulatory blood pressure reduction with SGLT-2 inhibitors: dose-response meta-analysis and comparative evaluation with low-dose hydrochlorothiazide. Diabetes Care. 2019;42:693-700.

76. Kario K, Okada K, Kato M, et al. 24-hour blood pressure-lowering effect of an SGLT-2 inhibitor in patients with diabetes and uncontrolled nocturnal hypertension: results from the randomized, placebo-controlled SACRA study. Circulation. 2018;139(18):2089.

77. Kandler MR, Mah GT, Tejani AM, Stabler SN, Salzwedel DM. Hydralazine for essential hypertension. Cochrane Database Syst Rev. 2011;2011:CD004934.

78. Cohn JN, Archibald DG, Ziesche S, et al. Effect of vasodilator therapy on mortality in chronic congestive heart failure. Results of a Veterans Administration Cooperative Study. N Engl J Med. 1986;314:1547-52.

79. Carson P, Ziesche S, Johnson G, Cohn JN. Racial differences in response to therapy for heart failure: analysis of the vasodilator-heart failure trials. Vasodilator-Heart Failure Trial Study Group. J Card Fail. 1999;5:178-87.

80. Cohn JN, Ziesche S, Smith R, et al. Effect of the calcium antagonist felodipine as supplementary vasodilator therapy in patients with chronic heart failure treated with enalapril: V-HeFT III. Vasodilator-heart failure trial (V-HeFT) study group. Circulation. 1997;96:856-63.

81. Udelson JE, DeAbate CA, Berk M, et al. Effects of amlodipine on exercise tolerance, quality of life, and left ventricular function in patients with heart failure from left ventricular systolic dysfunction. Am Heart J. 2000;139:503-10.

82. Multicenter Diltiazem Postinfarction Trial Research G. The effect of diltiazem on mortality and reinfarction after myocardial infarction. N Engl J Med. 1988;319:385-92.

83. Ferreira JP, Butler J, Rossignol P, et al. Abnormalities of potassium in heart failure: JACC State-of-the-Art Review. J Am Coll Cardiol. 2020;75:2836-50.

84. Packham DK, Rasmussen HS, Lavin PT, et al. Sodium zirconium cyclosilicate in hyperkalemia. N Engl J Med. 2015;372:222-31.
85. Anker SD, Kosiborod M, Zannad F, et al. Maintenance of serum potassium with sodium zirconium cyclosilicate (ZS-9) in heart failure patients: results from a phase 3 randomized, double-blind, placebo-controlled trial. Eur J Heart Fail. 2015;17: 1050-6.

86. Weir MR, Bakris GL, Bushinsky DA, et al. Patiromer in patients with kidney disease and hyperkalemia receiving RAAS inhibitors. N Engl J Med. 2015;372: 211-21.

87. Uijl E, Mirabito Colafella KM, Sun Y, et al. Strong and sustained antihypertensive effect of small interfering RNA targeting liver angiotensinogen. Hypertension. 2019;73:1249-57.

88. Ren L, Colafella KMM, Bovee DM, Uijl E, Danser AHJ. Targeting angiotensinogen with RNA-based therapeutics. Curr Opin Nephrol Hypertens. 2020;29:180-9.

89. Raal FJ, Kallend D, Ray KK, et al. Inclisiran for the treatment of heterozygous familial hypercholesterolemia. N Engl J Med. 2020;382:1520-30.

90. Laffin LJ, Bakris GL. Catheter-based renal denervation for resistant hypertension: will it ever be ready for "prime time"? Am J Hypertens. 2017;30: 841-6.

91. Bhatt DL, Kandzari DE, O'Neill WW, et al. A controlled trial of renal denervation for resistant hypertension. N Engl J Med. 2014;370:1393-401.

92. Desch S, Okon T, Heinemann D, et al. Randomized sham-controlled trial of renal sympathetic denervation in mild resistant hypertension. Hypertension. 2015;65:1202-8.

93. Mathiassen ON, Vase $\mathrm{H}$, Bech JN, et al. Renal denervation in treatment-resistant essential hypertension. A randomized, SHAM-controlled, doubleblinded 24-h blood pressure-based trial. J Hypertens. 2016;34:1639-47.

94. Bohm M, Kario K, Kandzari DE, et al. Efficacy of catheter-based renal denervation in the absence of antihypertensive medications (SPYRAL HTN-OFF MED Pivotal): a multicentre, randomised, shamcontrolled trial. Lancet. 2020;395:1444-51.

95. Fukuta H, Goto T, Wakami K, Ohte N. Effects of catheter-based renal denervation on heart failure with reduced ejection fraction: a systematic review and meta-analysis. Heart Fail Rev. 2017;22:657-64.

96. Laffin LJ, Bakris GL. Hypertension and new treatment approaches targeting the sympathetic nervous system. Curr Opin Pharmacol. 2015;21:20-4. 
97. Zile MR, Lindenfeld J, Weaver FA, et al. Baroreflex activation therapy in patients with heart failure with reduced ejection fraction. J Am Coll Cardiol. 2020;76:1-13.
98. Spiering W, Williams B, Van der Heyden J, et al. Endovascular baroreflex amplification for resistant hypertension: a safety and proof-of-principle clinical study. Lancet. 2017;390:2655-61. 